\title{
Design and Implementation of Advanced Wireless Tongue Drive/ Operated System for Paralyzed, Disabled \& Quadriplegic Patients
}

\section{Abbas $U^{1^{*}}$, Shaikh $M Z^{2}$, Panhwer $A^{2}$, Turk $S^{1}$ and Kumar $\mathbf{N}^{1}$}

${ }^{1}$ Institute of Biomedical Engineering and Technology, Liaquat University of Medical and Health Sciences, Jamshoro, Sindh, Pakistan

${ }^{2}$ Mehran University of Engineering and Technology, Jamshoro, Sindh, Pakistan

"Corresponding author: Abbas U, Institute of Biomedical Engineering and Technology, Liaquat University of Medical \& Health Sciences, Jamshoro, Sindh, Pakistan, Tel: 92229213305; E-mail: uzairabbas33@gmail.com

Rec date: Mar 23, 2016; Acc date: Apr 18, 2016; Pub date: Apr 26, 2016

Copyright: () 2016 Abbas U, et al. This is an open-access article distributed under the terms of the Creative Commons Attribution License, which permits unrestricted use, distribution, and reproduction in any medium, provided the original author and source are credited.

\begin{abstract}
We have got evolved a Wi-Fi, noncontact, unobtrusive, tongue-operated assistive technology called the Tongue controlled device. The TCS affords humans with paralysis, Quadriplegic diseases, minimum or no motion capability of their higher limbs, lower limbs with an efficacious tool for computer access and environmental control. A small permanent magnet secured at the tongue by using implantation, piercing, or tissue adhesives is used as a tracer, the motion of that is detected by way of an array of magnetic sensors established on a headset outside the mouth or on an orthodontic brace interior sent wirelessly to microcontroller by using wireless transceiver. The microcontroller's outputs signals are wirelessly transmitted to an ultraportable personal digital assistant compact computer carried at the user's clothing or wheelchair and are processed to extract the person's instructions. The consumer can then use those commands to get right of entry to a personal digital assistant computer, manage a power wheelchair, prosthetics and home appliances or have interaction with his or her surroundings.
\end{abstract}

Most adult humans have 32 teeth, and several commands can be linked to a combination of teeth or tongue gestures, making the possibilities countless.

Keywords: Wi-Fi; Quadriplegic diseases; Magnetic field

\section{Introduction}

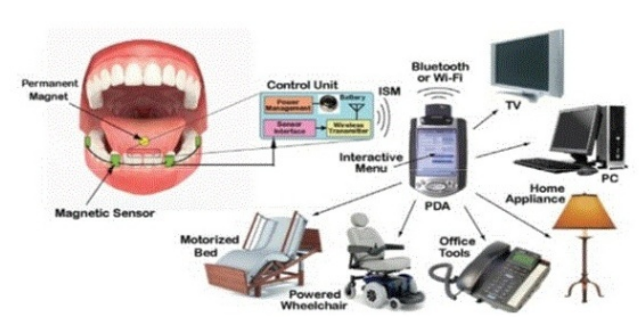

Figure 1: Illustrate the Tongue Drive Control System PC = personal computer; $\mathrm{PDA}=$ personal, Digital assistant $\mathrm{TV}=$ television.

Tongue has a set of unique characteristics that makes it a suitable appendage for manipulating paralyzed individuals' environments through the use of tongue-operated assistive devices. A number of these devices have been reviewed and a new one called Tongue Drive has been introduced Assistive Technology Tongue Drive system (TDS). The core TDS technology exploits the fact that even individual with severe paralysis that impairs limb movement, breathing and speech can still move their tongue and therefore, can fully utilize this extraordinary system. The device consists of a head set a compact computer and a tiny magnet attached to the tongue, simply movement of the tongue send the commands to the computer allowing users to steer their wheel chair operate their computers and generally control their environment in an independent way (Figure 1). We can continue to improve the system so, that it is not visible by reducing the size of the computer and replacing the headset needed to detect tongue signal with tiny sensors placed inside the user's mouth [1]

TDS consists of an array of magnetic sensors located inside the mouth, attached to the outer surface of the teeth on an orthodontic brace or outside of the mouth near the user's cheeks, mounted on a headset. The sensor array measures the magnetic field of a small permanent magnet, the size of a grain of rice, which is attached to the tongue by means of tissue adhesives. The sensor signals are digitized, and transmitted wirelessly to an external PC/PDA, which is worn by the user or attached to his/her bed or wheelchair. The received magnetic sensor array data is processed to determine the coordinates, orientation, and relative motion of the magnet with respect to the array of sensors in real time. This information is then used to control the movements of a cursor on the PC/PDA screen and to perform all other functions that an able-bodied individual can do with a mouse computer input device. The PC/PDA has a built-in wireless connection (Wi-Fi) potentially to a number of other devices, including a desktop computer, a phone, and a powered wheelchair in the user's environment. As a result, the user can access and control his/her environment through the PC/PDA interactive graphical user interface [2-7].

Before using the TDS, the user must train the system to link each designated tongue movement to an action. Training requires commands to be repeated several times for computer recognition, but is relatively fast. Each command is defined by collecting changes in the magnetic field in relation to the tongue position. The ultimate goal is to allow the user to designate every tooth to a command, Most adult humans have 32 teeth, and several commands can be linked to a 
combination of teeth or tongue gestures, making the possibilities countless. Through a wireless local area network (WLAN), the TDS can be linked to control household appliances, lights, locks, heating/air conditioning, etc. [7-10].

\section{Objectives}

Our objective or aim is to serve the Humanity every person have right to live their life happily and independently so we are making the Tongue Drive Control system for paralyzed, Quadriplegic and Disabled patients to control their environment easily like Wheel Chair, other appliances etc. and facilitate the life of these kinds of Patients.

The Disabled patients and patients with Spinal cord Diseases have difficult to perform daily life activities so this system help them to control their environment independently and perform their daily life activities. The device also could control prosthetic arms or legs. A prosthetic limb can have all the capabilities of a natural limb, but if you cannot control it, those capabilities are useless. "With the Tongue Drive System, the user can take advantage of all of the capabilities of the tongue to control his or her prosthesis and our aim is to make these kinds of patients work like normal peoples [11-13].

\section{Working Methodology}

In tongue controlling system a small magnet size of wheat placed on the tongue by using tissue adhesive force. The movement of this magnet varies magnetic field of magnet. The magnetic tracer detects variation in magnetic field. The network of magnetic sensors is placed on headset outside the mouth or orthodontic braces inside the mouth. The sensors output transmitted wirelessly personal digital assistant (PDA). The signal processor classifies and converts the sensors output to user commands (Figures 2 and 3 ). The advantage of tongue operated system is that the magnetic tracer gives lots of tongue movements, these movements provided with specific commands. We also set a standby mode command for sleeping, speaking and eating [14-16].

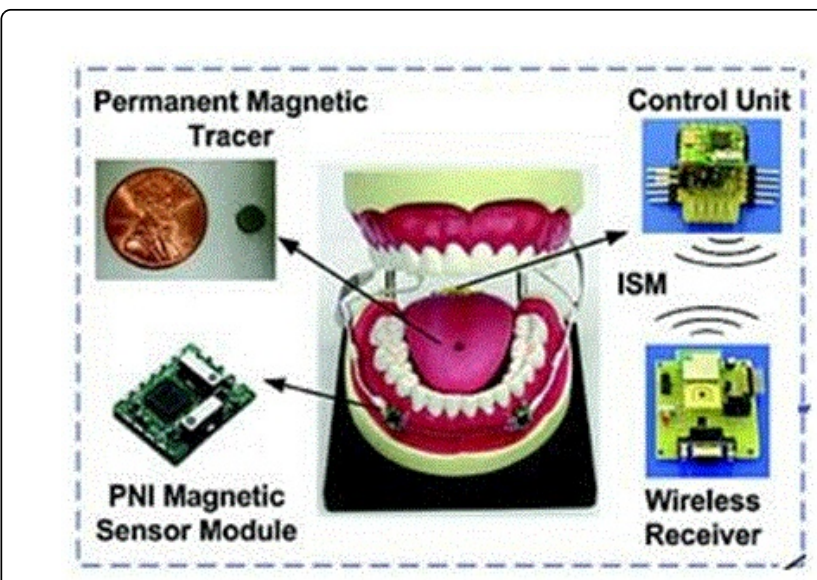

Figure 2: Components diagram of advanced wireless tongue drive/ operated system for paralyzed, disabled \& quadriplegic patients.

\section{Block Diagram}

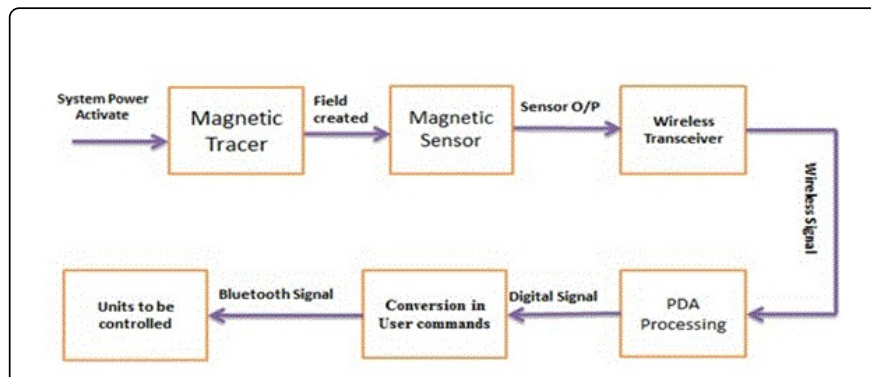

Figure 3: Block diagram of advanced wireless tongue drive/operated system for paralyzed, disabled \& quadriplegic patients.

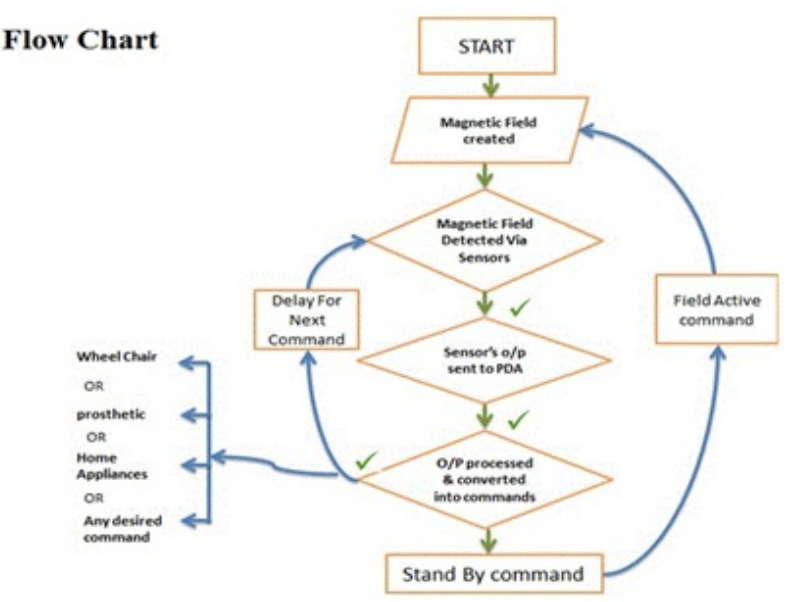

Figure 4: Flow chart of advanced wireless tongue drive/operated system for paralyzed, disabled \& quadriplegic patients.

\section{Prototype}

We have made prototype to facilitate the placement of the sensors. The function of prototype is to control the pointer of mouse with the help of tongue movements, there are six commands are given for the movement of pointer four commands for left, right, up, down and two commands single and double click (Figure 4). Four sensors are placed near to user's cheek whose specifications are given in following table. One sensor move cursor in $\mathrm{x}$-axis and other sensor in $\mathrm{y}$-axis and remaining two sensors move the cursor on $\mathrm{z}$-axis [16-18].

To minimize the interference of other magnetic field like earth's magnetic field, we use three axis modules as a reference. The three axis module output cancel out the interfering magnetic field (Table 1). All sensors output is received by ultra-low power microcontroller (MSP 430) the controller took eleven samples per second and activate only one module to reduce power consumption after collecting all data from all sensor were wirelessly send to personal computer (PC) through 2.4 GHz wireless link between two trans receiver (nRF2401). The whole system was powered by 3.3 volt button cell in a control unit [19-22]. 
Citation: $\quad$ Abbas U, Shaikh MZ, Panhwer A, Turk SK, Kumar N (2016) Design and Implementation of Advanced Wireless Tongue Drive/Operated System for Paralyzed, Disabled \& Quadriplegic Patients . J Bioeng Biomed Sci 6: 185. doi:10.4172/2155-9538.1000185

Page 3 of 5

\begin{tabular}{|l|l|}
\hline Specification & Value \\
\hline Control Unit & - \\
\hline Microcontroller & - \\
\hline Source and Type & $\begin{array}{l}\text { Texas Instruments; MSP430F1232 } \\
\text { Ultralow Power Microcontroller }\end{array}$ \\
\hline Dimensions & $22.5 \times 18 \times 16 \mathrm{~mm}^{3}$ \\
\hline Clock Frequency & $1 \mathrm{MHz}$ \\
\hline Sampling Rate & 11 sample/s/sensor \\
\hline Wireless Transceiver & - \\
\hline Source and Type & $\begin{array}{l}\text { Nordic Semiconductor; nRF2401single } \\
\text { chip } \\
2.4 \mathrm{GHz} \text { transceiver }\end{array}$ \\
\hline Dimensions & $15 \times 12 \times 3 \mathrm{~mm}^{3}$ \\
\hline Operating Voltage/Current & $2.2 \mathrm{~V} / \sim 4 \mathrm{~mA}$ \\
\hline Magnetic Sensor Module & - \\
\hline Source and Type & $\begin{array}{l}\text { PNl; magneto-inductive, MicroMag2, } \\
2-\text { Axis Magnetic Sensor Module }\end{array}$ \\
\hline Sensor Dimensions & $6.3 \times 2.3 \times 2.2 \mathrm{~mm}{ }^{3}$ \\
\hline Sensor Module Dimensions & $15 \times 12 \times 3 \mathrm{~mm}^{3}$ \\
\hline Resolution/Range & $0.015 \mu \mathrm{T} / 1100 \mu \mathrm{T}$ \\
\hline Inductance & 400 to $600 \mu \mathrm{H}$ at 100 kHz, 1 Vp-p \\
\hline Magnetic Tracer & - \\
\hline Source and Type & $\begin{array}{l}\text { RadioShack, Rare-Earth } \\
\text { Magnet } 64-1895\end{array}$ \\
\hline Size (Diameter $\times$ thickness) & $5 \mathrm{~mm} \times 1.3 \mathrm{~mm}$ \\
\hline Residual Magnetic Strength & $800 \mathrm{Gauss}$ \\
\hline
\end{tabular}

Table 1: Tongue drives system specifications.

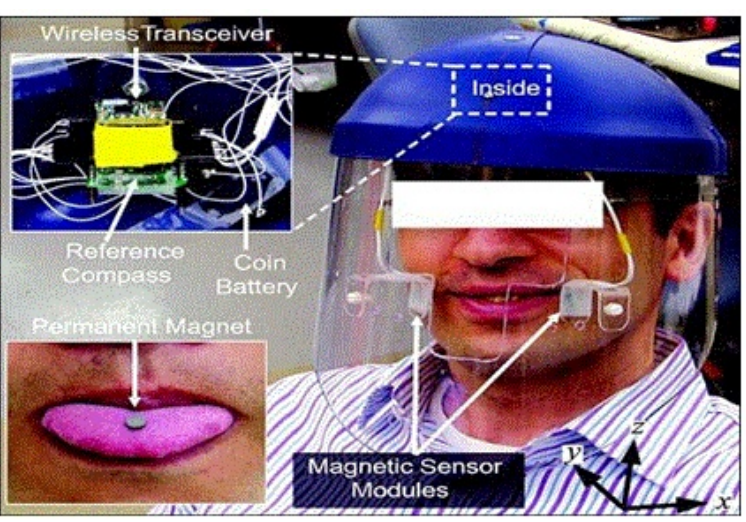

Figure 5: Prototype of tongue operated System for Human trials.

Magnetic racer: RadioShack, Rare-Earth Super Magnet is attached on tip of Tongue which is used to create the magnetic field.

Magnetic sensor: PNI Magnetic Sensor Module is used to detect the magnetic field created by magnetic tracer attached with tongue. Magnetic sensors are mounted on a headset outside the mouth or mounted on a dental retainer inside the mouth on teethes (Figure 5).

Wireless transceiver: Nordic Semiconductor; nRF2401single chip $2.4 \mathrm{GHz}$ transceiver is used wirelessly transmit the output of magnetic sensors to PDA using ISM band having frequency of $2.4 \mathrm{GHz}$ [23-25].

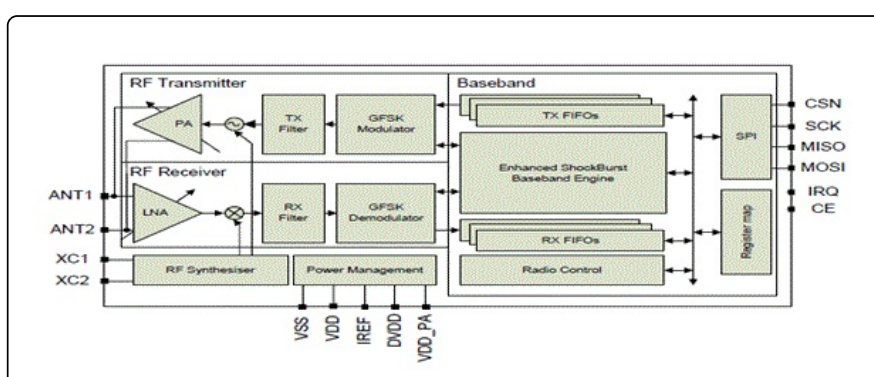

Figure 6: Block diagram of wireless transceiver.

Microcontroller: Texas Instruments MSP430F1232 Ultralow Power Microcontroller used for various applications. Ultralow-power mode is optimized to achieve extended battery life in different applications. It is a powerful 16-bit Microcontroller that provides maximum efficiency. The digitally well-ordered oscillator allows Micro controller to move from low power modes to active mode in less than $6 \mu$ s. The MSP430F1232 series are ultralow-power mixed signal microcontrollers with a built-in 16-bit timer, 10-bit A/D converter with integrated reference and data transfer controller and fourteen or twenty-two I/O pins (Figure 6). In addition, the MSP430F1232 series microcontrollers have built-in communication capability using asynchronous and synchronous protocols [25-28].

Personal digital assistant: PDA is compact computer used to process the signal and converts the signal into user's commands. Signal from PDA is transmitted via Bluetooth to control user's environment.

Reference compass: The reference compass is used to minimize the effects of external magnetic field interference, including the earth magnetic field. The reference compass was placed on top of the face shield so as to be far from the tongue magnet and to only detect the interfering magnetic field and the reference compass output was then used to predict and cancel out the interfering magnetic fields [1].

Battery: The entire system was powered by a $3.3 \mathrm{~V}$ coin sized battery which is placed together with the reference compass into the face shield cap.

\section{Sensor signal processing}

Sensor signal processing algorithm tool is develop in LABVIEW and MATLAB environments. In every twenty milli seconds three axial magnetic sensors provide data samples by measuring the magnetic field along three axes $(x, y, z)$ in real time sensor signal processing algorithm. A moving window picks 18 values of three samples of $x, y, z$ axis from 2 modules in 60milli seconds than data passes to three axis module compass to cancel out external magnetic fields i-e of earth than data reflect on virtual 3-D space using principle component analysis (PCA) than every tongue movement has specific position in 3-D (PCA) space. In order to identify six user defined position associated with specific command user is trained by moving tongue in six 
positions and by touching the teeth with the tip of tongue user repeat this task 10 times while SSP algorithm collects training samples and reflects these samples on PCA space and create a cluster of data on each commands we refer this section as training center (Figure 7). Every new incoming sample is reflected to PCA space. After finding the intended user command cursor move in given direction and user can hold his/her tongue in issued position that the pointer will move and reaches at certain velocity [1].

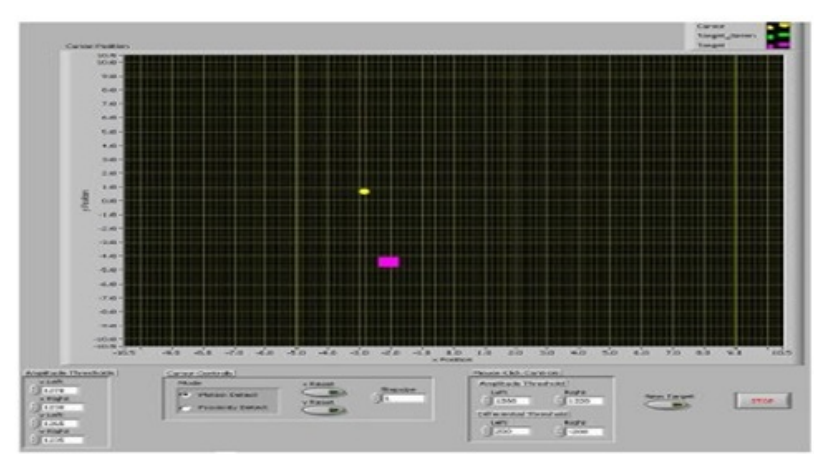

Figure 7: The Lab View developed prototype of tongue drive system.

In which rectangular pink mark is target in a random position, the small yellow circular dot is tracing the pink mark when magnet is held near to the sensor the yellow mark moves until it reaches on target.

Magnetic field creation: Magnetic field created by magnetic tracer inside mouth on the tongue which varied the magnetic field by Tongue movements.

Magnetic field detection: Those magnetic field variations detected by magnetic sensors mounted on a headset outside the mouth or mounted on a dental retainer inside the mouth on teethes.

Wireless transmission of sensor's output: Sensor's output is wirelessly transmitted via wireless transceiver (nRF2401) by using ISM (Industrial Scientific Medical) Band of $2.4 \mathrm{GHz}$ frequency to microcontroller.

Sending data to microcontroller: Texas Instruments; MSP430F1232 Ultralow Power Microcontroller is used to take the samples of data After reading all sensors, samples of data are wirelessly transmitted to a personal Digital Assistant across a $2.4 \mathrm{GHz}$ wireless link established between two identical nRF2401 transceivers.

Signal processing by PDA: PDA (Personal Digital Assistant) receives and processes the signal and converts the signal into user's commands.

Controlling different units: Signal is than transmitted through Bluetooth to different units to control the user's environment like Home Appliances, Wheel Chair, Prosthetics, and any other desired command.

Stand by command: We also set the stand by command for Sleeping, Eating, and Speaking and for other activities.

\section{Conclusion}

Advanced Wireless Tongue Drive/Operated System is beneficial which provides an immense help and support to Paralyzed, Disabled \& Quadriplegic Patients. Unfortunately; these patients life is void of many happiest moments of life. This system will help the Paralyzed,
Disabled \& Quadriplegic Patients to move and control their environment independently through the Signal Processing via sensor mechanism by carrying these tiny magnetic tracer and magnetic sensors. Ultimately; this system will result in serving the humanity which is indeed a greatest act. Our future work is to improve and enhance the quality of tongue controlled system and make the system smaller, faster and efficient. We add more commands to control the patient's environment [1].

\section{References}

1. Johnson AN, Huo X, Ghovanloo M, Shinohara M (2012) Dual-task motor performance with a tongue-operated assistive technology compared with hand operations. J Neu Eng and Rehab 9: 1-16.

2. Padmalatha E, Reddy RR (2011) Tongue Drive System to operate Computers- A New Tongue operated Assistive Technology. J Compr Appl Res and Devlp 1: 8-18.

3. Youssef B, Huo X, Ghovanloo M (2011) Control Preliminary Assessment of Tongue Drive System in Medium Term Usage for Computer Access and Wheelchair. Conf Proc IEEE Eng Med Biol Soc, Boston.

4. Huo X, Ghovanloo M (2010) Evaluation of a wireless wearable tongue computer interface by individuals with high level spinal cord injuries. J Neur Eng 7.

5. Huo X, Ghovanloo M (2009) Using unconstrained tongue motion as an alternative control surface for wheeled mobility. IEEE Trans on Biomed Eng 56: 1719-1726.

6. Huo X, Wang J, Ghovanloo M (2008) Introduction and Preliminary evaluation of Tongue drive system Wireless tongue operated assistive technology for people with little or no Upper limb function. J Rehab Res Develp 45: 921-930.

7. Huo X, Wang J, Ghovanloo M (2008) A magneto-inductive sensor based wireless tongue- computer interface. IEEE Trans Neural Syst Rehabil Eng 45: 921-30.

8. Jain M, Joshi H (2014) Tongue Operated Wheelchair for Physically Disabled People. IJLTET 4: 79-86.

9. Huo X, Wang J, Ghovanloo M (2007) A magnetic wireless tongue computer interface. 3rd Int IEEE/EMBS Conf Neu Eng Kohala Coast, New York.

10. Huo X, Wang J, Ghovanloo M (2007) Use of tongue movements as a substitute for arm and hand functions in people with severe disabilities. RESNA Annual Conf Phoenix, Arlington.

11. Krishnamurthy G, Ghovanloo M (2006) Tongue drive: A tongue operated magnetic sensor based wireless assistive technology for people with severe disabilities. IEEE Internl Symp Circ Syst Kos, New York.

12. Kim J, Park H, Bruce J, Sutton E, Rowles D, et al. (2013) The Tongue Enables Computer and Wheelchair Control for People with Spinal Cord Injury. Sci Transl Med 5.

13. Kim J, Huo X, Ghovanloo M (2010) Wireless Control of Smartphones with Tongue Motion Using Tongue Drive Assistive Technology. Conf Proc IEEE Eng Med Biol Soc 5250-5253.

14. Cohen A (1988) Biomedical signal processing. Boca Raton (FL): CRC Press 2ndEd New York.

15. Salem C, Zhai S (1997) An Isometric tongue pointing device.ACM SIGCHI Conf Hum Fact in Compt Systs New York.

16. Anderson KD (2004) Targeting recovery Priorities of the spinal cordinjured population. J Neuro trauma 21: 1371-1383.

17. Kandel ER, Schwartz JH, Jessell TM (2000) Principles of Neural Science. Jordan Uni Sci Tech Fac Eng Biomed Eng Dept, New York.

18. Oliver RG, Evans SP (1986) Tongue size, oral cavity size, and speech. The Angle Orthodontist 56: 234-243.

19. Wolfgang N, Christian A, Silvio N, Gerhard S (1998) Tongue-mouse for quadriplegics. J Micmecha Mic-eng 8: 155-157. 
Citation: $\quad$ Abbas U, Shaikh MZ, Panhwer A, Turk SK, Kumar N (2016) Design and Implementation of Advanced Wireless Tongue Drive/Operated System for Paralyzed, Disabled \& Quadriplegic Patients . J Bioeng Biomed Sci 6: 185. doi:10.4172/2155-9538.1000185

Page 5 of 5

20. Struijk LNSA (2006) An inductive tongue computer interface for control of computers and assistive devices. IEEE Trans Biomed Eng 53: 25942597.

21. Anderson KD (2004) Targeting recovery priorities of the spinal cord injured Population. J Neurotrauma 21: 1371-1383.

22. PNI MS 21002 Axis Magnetic Sensor Specifications.

23. Chee YH, Rabaey JM, Niknejad A (2006) Ultra-Low Power Transmitters for Wireless Sensor Networks. University of California, Berkeley.
24. The UAB-SCIMS Information Network, UAB Spinal cord injury Model System.

25. Spinal Cord Injury: Hope Through Research, NIH.

26. Tongue Touch Keypad ${ }^{\mathrm{m}}$.

27. Jouse2, Compusult Limited.

28. USB Integra Mouse, Tash Inc. 